\title{
Some considerations about a report on 'Public health in an era of genomic-based and personalized medicine' from the Public Health Foundation, Cambridge
}

\author{
Jean-Jacques Cassiman
}

Received: 27 January 2011 / Accepted: 4 February 2011 /Published online: 19 February 2011

(C) Springer-Verlag 2011

The Public Health Foundation, Cambridge UK, released in November 2010 a report entitled: 'Public health in an era of genome-based and personalized medicine'. The report (Hall 2010 ) is based on a meeting convened at Ickworth House in May in Suffolk, UK and was attended by a group of 24 international and multidisciplinary experts. In the 35 pages report, five issues relevant for public health genomics (PHG) are discussed. The report concludes with six recommendations for future global public health practice. The style of the report is clear, and a short conclusion for each issue is presented in separate boxes.

It is unquestionable that in time PHG could gradually revolutionize medical practice. The report therefore provides a series of important answers to questions which will need to be resolved before PHG can be safely introduced in public health programmes. As can be expected, however, the time available and the different opinions on a series of issues by believers and non-believers in PHG did not allow one to come to too many concrete proposals.

Of course one can only agree with most of the conclusions and recommendations, but a number of issues could have been elaborated a bit more extensively.

To give just a few as examples:

- On the topic of the potential for PHG, it is good to list a series of shortcomings and to draw attention to the over enthusiasm that was initially generated when this field was first brought to the attention in the statement of the Bellagio meeting in 2005 (see reference for report).

J.-J. Cassiman $(\bowtie)$

Centre for Human Genetics, Catholic University of Leuven,

Kapucijnenvoer 33,

3000 Leuven, Belgium

e-mail: Jean-jacques.cassiman@med.kuleuven.be
What needs to be done to return to the real word is proposed in the report, but how it has to be concretely realized is not very detailed or explicit; nevertheless, it is time to become specific and clear suggestions on what needs to be done are required.

- Most geneticists would agree that genetic exceptionalism needs to be banned. Nevertheless, this should not lead to banning genetics or the geneticists from the implementation of genomics. Indeed in the coming years the diseases which will be the first to provide a model on how genomics can be adequately introduced in clinical practice will be mainly Mendelian diseases. When the knowledge about more common diseases will be applicable in practice, it is clear that here also geneticists, in constructive interaction with other specialties, will have to play an important role. Indeed obtaining a good family history, determining and explaining the risk for the next generations to develop similar diseases- the transgenerational aspects - are unique expertises of the geneticists (Kosztolanyi and Cassiman 2010).

- Appropriate informed consent is indeed an important issue. But except for stating that this needs to be solved, few clues are given on how this could be tackled and what elements should be included in such consent form.

- Regarding the need for motivating a change in behaviour of the patients, a correct precondition to have an impact on public health, one should also find ways of improving therapy adherence (compliance) responsible for the numerous failures of medical treatment and preventive measures which could undermine the potential positive effects of PHG.

- The whole population should indeed benefit from PHG strategies. A major obstacle to this laudable aim will be whether an appropriate health care system (infrastruc- 
ture, expertise and health insurance) exists. We should not underestimate this and jump directly to the implementation of genomics. Not only low and middle income countries might have difficulties with this. The situation of the health care system in the USA, illustrates that even rich countries might have problems introducing PHG strategies in a just and social way.

In view of the potential importance of PHG, some additional considerations are formulated-philosophical, technological or even practical - which were not or only briefly discussed in the report, but might need to be considered in future meetings.

- A series of fundamental questions need to be answered, such as: what is the ultimate aim of these PHG strategies. Of course we all want help in curing or controlling all major diseases, but how far do we want to go in this? Do we focus only on serious diseases or on treatable or preventable diseases? Will a threshold be decided for the risk to develop diseases at which prevention will be required or even becomes compulsory? Will intensive application of PHG strategies lead to excessive medicalization/geneticalization of the population? Public health is different from wellbeing. Could a conflict in time develop between these two important aspects of life and of society? Can a medical approach alone guarantee well-being in a society? How can we find this equilibrium between improving health and maintaining or increase wellbeing by doing so?

- PHG is of course aimed at improving public health. The risk nevertheless exists, as our knowledge increases about what makes us sick, that we also learn more about how our normal characteristics are determined. The boundary between health and disease may start fading as a result. Genetic and environmental causes of the variations in normal characteristics might receive much more attention and ultimately people might become more interested in how to influence/select 'normal' traits. The money spent on plastic surgery in western countries gives a good indication that the public confuses-rightly or wrongly - health with well-being. The risk to develop a particular disease later in life might indeed not be the greatest concern of our populations.

- The technological revolution makes predictions about which instruments or approaches will in time become the preferred tools/methods to analyse genomes and genome products (RNA, proteins, metabolites...) almost impossible. Today's market leader may be rapidly replaced by another temporary leader. To be able to cover the necessary investments and improve the efficiency of the services, the chances are that larger reference centres with appropriate diversified technological platforms will be set up responsible for the high throughput analysis of thousands of samples a year. Local clinical services would then mainly serve as entry point for the patient and interpretation of his/her testing results. In fact this is somehow what the direct to consumer (DTC) services tried to set up. We should actually be grateful to the DTC companies that we were forced to review this new approach as well as its potential impact on our services and on the population. The questions to be answered in this regard will be: what service provision will be optimal in the future? What will be the role of the geneticists in this? How can we convince the policy makers to follow our suggestions? Should we plan an orderly introduction of these services or wait and see what happens, let market forces decide?

- Impressive efforts are underway to identify tissue-, organ- and individual-specific networks of interacting proteins (Barabasi et al 2011). Rather than the symptom-based approach we have today, they will undoubtedly become the basis on which diseases and 'diseasomes' will be identified in the future. Moreover, they will allow one to measure the effect of genetic polymorphisms and of epigenetic and environmental influences on the function of these networks and give a solid scientific basis for 'personalized (stratified groups) medicine'. In addition, they will be the basis on which new treatments will be designed. The available knowledge about these networks can in most cases not yet be used in medical practice. Also in model organisms the role of the 'dark genome' - the non-coding part of our genome-is being successfully unraveled and opportunities to do the same for humans are becoming available (Blaxter 2010, Davidson 2010). More informationtime and research - is needed before the knowledge will be applicable in the clinic, but will we be able to wait? In this regard, as stated in the report, proven clinical validity and utility of the research findings as well as the ethical, legal and societal aspects will be evaluated before their clinical application can be considered. This will require a fundamental change in the regulations about genetic/medical testing. The IVD directive of the $\mathrm{EU}$ is under revision. Even in its new formulation, it may not provide sufficient regulation to guarantee that all tests done in academic or private settings in the EU are done under appropriate quality criteria. Moreover, it will probably not be able to regulate tests offered over the internet. Many tests used today have not been validated for clinical validity, let alone clinical utility. The laudable efforts of EGAPP (Teutsch et al. 2009) to review a small number of potential genomic tests illustrate how difficult and time-consuming this is. Even a global system to review new tests will require 
years before it will be able to gather sufficient data allowing a thorough evaluation (Grimaldi et al 2010). If on the other hand new tests would be submitted to the same scrutiny as those to which drugs are submitted (clinical trials) before being allowed in practice, it would raise the cost of such tests to unaffordable levels and would unnecessarily delay their use. Possibly, the conditional introduction of tests, as is proposed in some countries for orphan drugs, might allow a controlled entry into practice, with appropriate revision and decision on its further use, after a number of years.

In conclusion, the report of this interesting meeting has listed in more detail than before what the way forward is. Up to specific groups in the different continents to start defining concrete measures, as has already been done for some aspects by the EU funded PHGEN project (see website), and which will continue in the ongoing PHGENII. In addition, one should not shy away from trying to answer more fundamental societal questions about the impact of PHG in the long run. Only then will the different stakeholders know how PHG can be applied to really improve the health and well-being of our population.

\section{References}

Barabàsi A, Gulbahce N, Loscalzo J (2011) Network medicine: a network-based approach to human disease. Nat Rev Genet 12:56-68

Blaxter M (2010) Revealing the dark matter of the genome. Science 330:1758-1759

Davidson EH (2010) Emerging properties of animal gene regulatory networks. Nature 468:911-920

Grimaldi KA, Look MP, Scioli GA, Clavero JC, Marinos S, Tagaris T (2010) Personal genetics: regulatory framework in Europe from a service provider's perspective. Eur J Hum Genet. doi:10.1038/ ejhg. 2010.189

Hall A (2010) Public health in an era of genome-based and personalised medicine www.phgfoundation.org

Kosztolányi GY, Cassiman J-J (2010) The medical geneticist as expert in the transgenerational and developmental aspects of diseases. Eur J Hum Genet 18:1075-1076

Genome-based Reseach and Popukation Health. Report of an expert workshop held at the Rockefeller Foundation Study and Conference Centre, Bellagio, Italy, 14 -230 April 2005. Available at http://dceg.cancer.gov/files/genomicscourse/bellagio-011807.pdf

PHGEN www.phgen.eu

Teutsch SM, Bradley LA, Palomaki GE, Haddow JE, Piper M, Caloge N, Dotson WD, Douglas MP, Berg AO (2009) The Evaluation of Genomic Applications in Practice and Prevention (EGAPP) initiative: methods of the EGAPP Working Group. Genetics in Medicine 11:3-14 www.egapppreviews.org 